\title{
Transforming an Academic Radiochemistry Facility for Positron Emission Tomography Drug cGMP Compliance
}

\author{
Shaojun Zhu $\odot,{ }^{1}$ Sherly Mosessian, ${ }^{2}$ Kurt Kroeger, ${ }^{3}$ Saman Sadeghi, ${ }^{3}$ Roger Slavik, ${ }^{1}$ \\ Simon Kinloch, ${ }^{4}$ Melissa Moore,${ }^{5}$ Martin Allen-Auerbach, ${ }^{1}$ Johannes Czernin, ${ }^{1}$ \\ Michael Phelps ${ }^{3}$ \\ ${ }^{1}$ Ahmanson Translational Theranostics Division, Department of Molecular and Medical Pharmacology, UCLA, Los Angeles, CA, 90095, \\ USA \\ ${ }^{2}$ Digital Technology (DGIT), David Geffen School of Medicine Dean's Office, UCLA, Los Angeles, CA, 90095, USA \\ ${ }^{3}$ Department of Molecular and Medical Pharmacology, UCLA, Los Angeles, CA, 90095, USA \\ ${ }^{4}$ Proceutical Ltd, Stroud, UK \\ ${ }^{5}$ SOFIE, Inc, Culver City, CA, 90230, USA
}

\begin{abstract}
In light of the United States Food and Drug Administration (FDA) requirement of 21 CFR 212 current Good Manufacturing Practice (cGMP) for FDA-approved position emission tomography (PET) drugs, the University of California Los Angeles (UCLA) Biomedical Cyclotron (BMC) transformed from a pre-cGMP era academic cyclotron and radiochemistry facility to a current cGMP-compliant PET drug manufacturer. In this article, we share the financial and regulatory compliance aspects of the "transformation" required to develop a sustainable quality system to support the production of two PET drugs under Abbreviated New Drug Applications (ANDAs).
\end{abstract}

Key words: cGMP, FDA, PET drugs, Compliant, Transformation, Academic

\section{Introduction}

Positron emission tomography (PET) is a non-invasive molecular imaging technique that images the biology of disease throughout the body. PET imaging involves the administration of a PET drug, the production of which is regulated in the USA by the Food and Drug Administration (FDA) since the FDA Modernization Act (FDAMA) in 1997 [1]. Before the FDAMA, PET drug preparation was considered a "practice of medicine and pharmacy" under United States Pharmacopeia (USP) chapters 823 and

Shaojun Zhu and Sherly Mosessian contributed equally to this work. Kurt Kroeger is deceased. This paper is dedicated to his memory.

Correspondence to: Shaojun Zhu; e-mail: SZhu@mednet.ucla.edu
797. As a mandate by FDAMA, FDA issued 21 CFR 212 current Good Manufacturing Practice (cGMP) regulations for PET drugs on December 10, 2009 [2], as well as the cGMP guidance document for PET drugs [3]. The PET community was given 2 years to bring PET drug manufacturing to cGMP compliance. The original enforcement date was December 12, 2011, which was later extended for an additional 6 months [4].

PET cGMP regulations apply to marketed PET drugs in the USA under approved New Drug Applications (NDAs) or Abbreviated New Drug Applications (ANDAs). Investigational PET drugs can choose to follow either PET cGMP or USP chapter 823. Manufacturing requirements for therapeutic radiopharmaceuticals (also known as radiotherapeutics) is governed by the traditional cGMP regulations contained in 21 CFR 210 and 211. 
The UCLA Ahmanson Biomedical Cyclotron and Radiochemistry Facility (BMC) was first established in the 1970s. In 1990 and 1994, two CTI/Siemens RDS-112 cyclotrons were commissioned. These cyclotrons accelerate negative ion protons to an energy of $11 \mathrm{MeV}$ for the production of curie levels of fluorine-18 and other radioisotopes. Prior to any modification for compliance with PET drug cGMP, the UCLA BMC was fully equipped with the required Quality Control (QC) equipment to perform dose calibration, highperformance liquid chromatography (HPLC), gas chromatography (GC), radio-thin layer chromatography (radioTLC), gamma spectrometry, and other PET drug analytical testing.

The two cyclotrons, internally named RDS1 and RDS2, are housed in two separate areas within the same building, together creating the BMC facility. The RDS1 area is dedicated to 2-deoxy-2-[ $\left.{ }^{18} \mathrm{~F}\right]$ fluoro-D-glucose ( $\left.\left[{ }^{18} \mathrm{~F}\right] \mathrm{FDG}\right)$ and $\left[{ }^{13} \mathrm{~N}\right]$ ammonia $\left(\left[{ }^{13} \mathrm{~N}\right] \mathrm{NH}_{3}\right)$ production for clinical service. The RDS2 area is dedicated to supporting clinical and preclinical research of novel PET probes under effective Investigational New Drugs (INDs), Radioactive Drug Research Committee (RDRC) protocols, or UCLA Animal Research Committee (ARC) protocols. In addition, BMC runs a basic radiochemistry research program in the RDS2 area.

In 2011, the BMC established PET drug cGMP manufacturing capabilities and filed two ANDA applications with the FDA for $\left[{ }^{18} \mathrm{~F}\right] \mathrm{FDG}$ and $\left[{ }^{13} \mathrm{~N}\right] \mathrm{NH}_{3}$. In 2012, the BMC was inspected by the FDA for pre-approval inspection (PAI), with subsequent approval for both ANDA applications.

In this article, we share our experience of bringing an academic PET manufacturing site to cGMP compliance and maintaining cGMP, as well as the financial aspect of the PET cGMP operation at the UCLA BMC.

\section{Approach}

\section{Establishing cGMP at BMC}

To establish a PET cGMP environment at the UCLA BMC for regulatory compliance, the BMC recognized the following prerequisites:

- A leadership team committed to ensuring that there are adequate resources to support the development and maintenance of the Quality System (QS) and the quality culture of the operation

- A robust and appropriate QS for PET drug manufacturing

- Production facilities, laboratories, and equipment qualified for PET cGMP manufacturing and analysis

- Appropriate qualified manufacturing and QC equipment

- Validated manufacturing processes and QC methods

- Trained professional staff
To assess whether the BMC had these in place and complied with the FDA cGMP guidance document, the BMC management team engaged an experienced quality consulting company from the commercial radiopharmaceutical manufacturing sector, to advise on how to become "inspection ready" for the FDA pre-approval audit.

Gap Analysis Process The consultant analyzed the BMC cGMP systems using a gap assessment checklist based on 21 CFR 212 (Supplement online: Gap analysis checklist) to record information and provide a road map identifying areas where improvements could be made in preparation for an upcoming inspection. Following this review, BMC management devised a Management Action Plan to close the gaps prior to the FDA inspection. Management monitored the project's progress and, on completion, the operation was reassessed to confirm that the gaps were closed. Shortly after completion of the gap closure project, the FDA contacted the $\mathrm{BMC}$ to schedule a pre-approval inspection. Both PET probes $\left[{ }^{18} \mathrm{~F}\right] \mathrm{FDG}$ and $\left[{ }^{13} \mathrm{~N}\right] \mathrm{NH}_{3}$ received "recommended for approval" from the inspection with only minor FDA Form 483 observations of deficiencies issued.

The operation has since been regularly re-assessed by scheduled quality system review and internal audits to ensure continuous compliance and quality in PET drug production. Regular ongoing reassessment also includes addressing new requirements introduced since the last analysis, thus establishing a system for continuous improvement of the operation.

Facilities and Equipment Improvements to Meet 21 CFR 212 Environmental classification of an ISO 5 (class 100) environment for aseptic manipulation (such as product vial assembling, QC sample withdrawal, sterility test inoculation, etc.) is required by cGMP guidance from the FDA. Two laminar air flow hoods (LAFH, Type II A Biosafety Cabinet) were acquired and installed in production and QC rooms, respectively. The locations of the hoods were selected to ensure optimal workflow and minimal traffic. The LAFH installed in the production room is lead shielded for the safe handling of radioactive samples, which is considered an appropriate engineering control for the amount of radioactivity BMC handles. The lead shield minimizes the operator's exposure when withdrawing samples for QC. It contains an integrated dose calibrator to measure End of Synthesis (EOS) dose activity. The second LAFH installed in the QC room is not shielded and is mainly used for non-radioactive operations, such as assembling product vials and inoculating sufficiently decayed QC samples into growth media for sterility testing. Both LAFHs were ISO 5 certified initially and tested thereafter for conformity every 6 months, including inflow and downflow velocity measurements, smoke test, HEPA filter integrity testing, and particle counts. Moreover, all aseptic operations were environmentally monitored by air settle plates, contact 
plates, and touch plates, according to the FDA PET cGMP guidance. UCLA BMC chose to perform sterility testing inhouse. Although this task could be outsourced to a qualified analytical testing company, it should be noted that outsourcing brings additional expense and work auditing the company for compliance. Figure 1 illustrates the floor plan and material/personnel flow inside the BMC production/QC area.

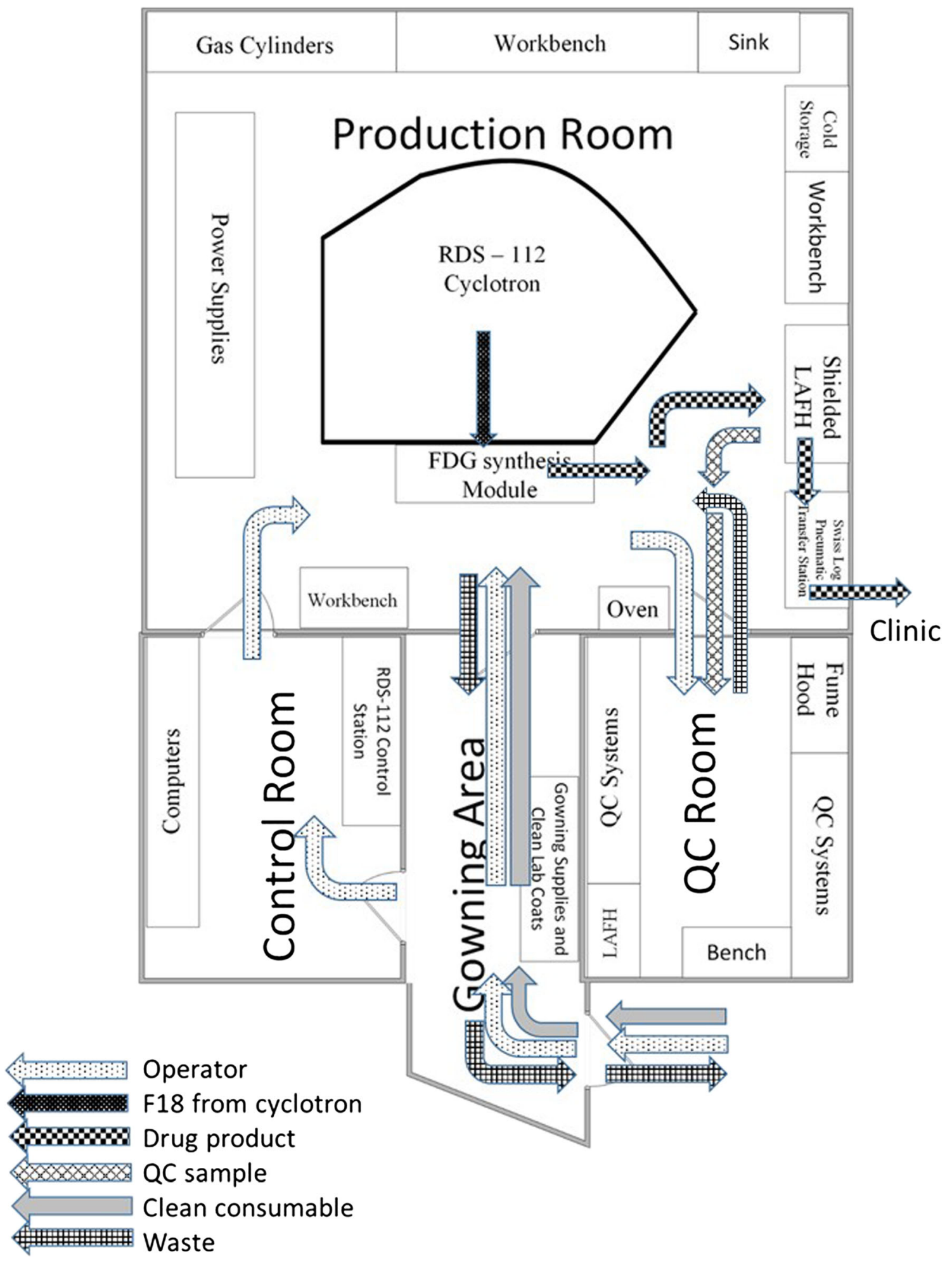

Fig. 1. BMC floor plan and material/personnel flow. 
Staff Training and Familiarization with 21 CFR 212 The BMC conducted an initial PET cGMP and microbiology training for staff by external consultants, who were selected as highly regarded experts in the field. All staff demonstrated proficiency in PET cGMP and aseptic handling by passing written assessment after the training. They were also observed performing tasks by quality assurance specialists for compliance.

Management of Documentation As advised by our quality consultant, we outlined the hierarchy of cGMP documentation to be established (Fig. 2).

The top tier documentation included a site master file and quality system manual. These top tier documents outlined the quality commitment and responsibility oversight at the BMC. The second tier documentation included detailed Quality Assurance (QA) procedures, such as a validation plan, change control, personnel training policy, finished product release, investigation of out-of-specification (OOS), out-of-trend or process deviation, product recall, complaint handling, corrective and preventive actions (CAPAs), and conditional release procedures. The third tier of documentation contained material/component specification sheets and standard operating procedures (SOPs) for manufacturing, QC testing, and equipment calibration/maintenance. The fourth tier consisted of records of performed tasks and production/QC activities.

Management of Materials The acceptance criteria for materials used in routine productions and quality control procedures were established and approved in written Specification Bulletins (SB). All incoming materials and components were quarantined upon receipt and underwent acceptance/rejection procedures outlined in the corresponding SBs. Subsequently, the accepted materials were affixed with acceptance labels and tracked by assigned, unique inventory tracking numbers (ITN). Any in-house prepared solutions or reagents were tracked by unique preparatory tracking numbers (PTN). BMC has an off-site material storage room in close proximity, which is equipped with a temperature-controlled freezer and refrigerator monitored by 21 CFR part 11 compliant Wi-Fi-enabled digital temperature probes. Only accepted and properly tracked materials can be brought into production/QC areas.

Process Validation and Operator Qualifications Process validation runs and final product stability testing were performed and documented for all PET drugs. The final products underwent stability testing at the EOS and proposed expiry time. Final product vials were stored in an upside-down fashion at room temperature to mimic the worst-case scenario during shipment. Each radiochemist passed three consecutive qualification runs and three initial media fill simulations to qualify as an operator.

QC Test Equipment and Methods The BMC utilized the existing analytical equipment for quality control tests. This equipment consisted of dose calibrator(s), GC, HPLC, radioTLC, etc., all of which had undergone a performance qualification process. Compendial analytical methods were verified for suitability, while non-compendial methods underwent a validation process. Documentation of the aforementioned activities was archived in the BMC document room. During routine productions, day of use instrument check or system suitability tests were performed on a continual basis. It is important that QC

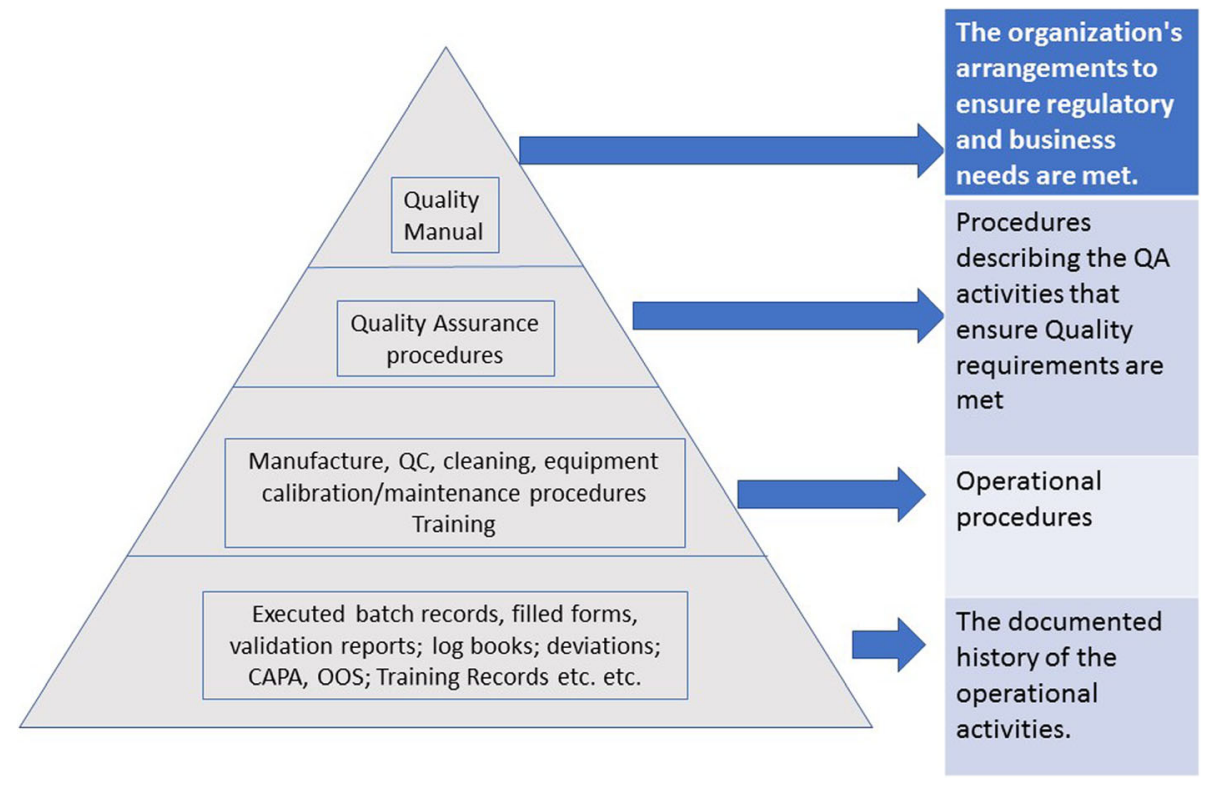

Fig. 2. PET cGMP documentation hierarchy at BMC. 
equipment pass the testing acceptance criteria before being used for analytical testing.

\section{Maintaining $c G M P$ at $B M C$}

After establishing the PET cGMP environment at BMC, we recognized the maintenance and continuous selfimprovement of the PET cGMP as the most challenging task. A QA program was established at the BMC to ensure continuous cGMP compliance. The key elements of the QA program are listed below:

QA Meetings Periodic QA staff meetings and QA management meetings are essential to the QA program at the BMC. These meetings are to discuss and address qualityrelated issues and concerns of the entire operation, including facility, equipment, materials, production, and analytical methods.

Facility and Equipment Routine facility cleaning and upkeep, equipment check, cleaning, preventive maintenance, and calibration are performed and documented at preset intervals. The environmental classifications of production area, QC area, and ISO 5 LAFH are routinely monitored and certified by an outside contract vendor.

Change Control Change controls are performed when any production or QC-related material/equipment/method change is requested. Risk assessment for the potential impact to product quality is evaluated and documented. Additional verification or validation data are generated for change control. Any material, process, and QC testing changes are filed with the FDA based on their significance categories (major, moderate, or minor).

Periodic Quality Indicator Testing and Stability Testing Periodic quality indicator testing (PQIT) and annual final product stability testing are conducted as part of post marketing commitment. PQIT varies between different products, but in general, it includes radionuclide purity testing to detect long-lived radionuclide impurities, specific activity (if applicable), and other potential chemical impurities (such as 2-chloro-2-deoxy-D-glucose for $\left.\left[{ }^{18} \mathrm{~F}\right] \mathrm{FDG}\right)$.

Documentation Review Periodic document/record review is a key component of continuous compliance. Every day, the BMC generates records such as executed batch records, material/reagent tracking, product vial assembly, production and QC raw data, etc. Periodic review adds assurance that all records are completed and filed correctly, and that SOPs contain clear instructions. At the $\mathrm{BMC}$, production and $\mathrm{QC}$ records are usually reviewed quarterly before being archived. Quality assurance and operational procedures are reviewed every 3 years at minimum.

Internal Audit An internal cGMP audit is conducted annually by a qualified QA person or a contracted external consultant. Any problems or inconsistencies discovered during the audit are followed up for correction or improvement. Identified corrective or preventive actions are implemented and subsequently verified through the CAPA program at BMC.

Personnel Training, Qualification, and Re-qualification cGMP training and radiochemist re-qualification are offered on an annual basis. Annual cGMP refresher trainings are coordinated with the clinic schedule to ensure radiochemists have undivided attention during the training. In addition, qualified radiochemists are requalified annually by passing one medial fill simulation to ensure proper aseptic technique.

Investigations and Corrective and Preventive Actions Product batches that are out-of-specifications, out-oftrend, or with a yield that exceeds action limit mandate a full investigation. During the investigation, root cause analysis and identification, risk assessment, and corrective/ preventive actions (identification/implementation/verification) are performed and documented.

Complaint Handling Written procedures for complaint handling are established at BMC in case a drug quality or drug adverse experience-related complaint arises.

Regulatory Lifecycle Finally, lifecycle management and regulatory filing are fulfilled as a continuous effort during the lifetime of the marketed products. As mentioned before, this usually includes an annual establishment registration and labeler code update, as well as annual Generic Drug User Fee Act (GDUFA) establishment self-identification. Annual reports with minor $\mathrm{CMC}$ changes are filed within 60 days of anniversary dates. Any moderate to major CMC change needs an ANDA supplement filing for either Change to be Effective 0/30 days (CBE0/30) or Prior Approval Supplement (PAS). In case of sterility failure, a Field Alert Report (FARs) is required to be filed within 3 days to identify quality defects in distributed drug products. Any known post marketing adverse drug experiences require reporting to the FDA. 


\section{Financial Model}

\section{Cost for Establishing PET cGMP at BMC}

To meet the cGMP requirements of December 2011, the UCLA Department of Molecular and Medical Pharmacology (DMMP), which uniquely contains the $\mathrm{BMC}$ and the Nuclear Medicine and PET/CT clinical service, implemented changes to bring the facility and its radiopharmaceutical production practices in compliance with the new regulations. The financial impact of these changes was around $\$ 855,000$ and details are included in Table 1.

Equipment The facility contained sufficient and appropriate equipment to meet the requirements. The DMMP obtained an additional lead-shielded laminar flow hood dedicated to aseptic product sampling for QC analysis.

Dedicated Rooms The department expanded the existing facility by two rooms: one as the document storage room and another as the supply room containing cold, dry, acid, base, and flammable containers.

Regulatory The department acquired the expertise of a regulatory consulting company to assist in reviewing, assembling, and electronically submitting two ANDA applications: $\left[{ }^{18} \mathrm{~F}\right] \mathrm{FDG}$ and $\left[{ }^{13} \mathrm{~N}\right] \mathrm{NH}_{3}$. In addition, investments were made to obtain appropriate cGMP training for the staff and utilize the expertise of a PET quality consulting company to provide a gap analysis and identify areas that required improvement.

Lost Revenue The BMC was run by a small number of staff with in-depth expertise of the entire operation. As a result, the staff were released from routine production duties to assist with producing necessary data and standard operating procedures to meet the cGMP and ANDA filing requirements.

The facility produced on average $\$ 100,000 /$ month in revenue from supplying $\left[{ }^{18} \mathrm{~F}\right] \mathrm{FDG}$ and $\left[{ }^{13} \mathrm{~N}\right] \mathrm{NH}_{3}$ for our clinical PET/CT service. The 4-month shut down of the operation resulted in a revenue loss of $\$ 400,000$.

Table 1. Financial impact to BMC for establishing PET cGMP

\begin{tabular}{ll}
\hline Description & Cost \\
\hline Purchase and install of additional laminar flow hood & $\$ 60,000$ \\
Lost revenue due to production pause & $\$ 400,000$ \\
Establishment of a document storage room & $\$ 50,000$ \\
Establishment of a dedicated supply room & $\$ 80,000$ \\
Regulatory cost for ANDA review and submission & $\$ 250,000$ \\
cGMP training for staff & $\$ 5000$ \\
Regulatory cost for gap analysis & $\$ 10,000$ \\
Total & $\$ 855,000$ \\
\hline
\end{tabular}

\section{Cost for Maintaining GMP at BMC}

Regulatory Registration and Lifecycle Management Cost As a PET drug manufacturer, the BMC is subject to a semiannual drug listing update (every June and December), an annual drug establishment registration (October 1 to December 31), and an annual GDUFA generic drug facility self-identification (May 1-31). The BMC handles these submissions in-house and thus does not incur any additional cost.

BMC holds two active ANDAs submitted in electronic Common Technical Document (eCTD) format. eCTD publishing and ESG submission is outsourced to a regulatory consulting company. The decision to outsource considers the high cost of in-house eCTD publishing setup and maintenance/user training, and its relatively low volume of usage. Minor Chemistry Manufacturing and Controls (CMC) changes that occurred after ANDA approval are reported in annual reports to the FDA. Based on the BMC's experience, the cost of eCTD publishing and submission service for two annual reports is around $\$ 8000$.

The cost of annual cGMP maintenance is included in Table 2 .

Staff Training Annual cGMP training is provided to the BMC staff members. The BMC sets training schedules every year by alternating between internal training and an external consultant. Internal training is conducted by senior quality assurance personnel, with updates from regulatory sessions held at the Society of Nuclear Medicine and Molecular Imaging (SNMMI) annual meeting. On average, the cost of training by an experienced domestic consultant is $\$ 4000$.

PQIT Testing, Stability Testing, and Personnel Re-qualifications As part of our ANDA post marketing commitment and quality system, stability testing and PQIT testing are conducted according to their preset schedules. The costs for post marketing commitment run at $\$ 3-5000$ annually.

New operational staff are trained by a mix of educational literature, classroom teaching, and supervised on the job training. To initially qualify in performing aseptic manufacturing, an operator must perform three consecutive and successful media fill simulations. Trained operational

Table 2. Cost of annual cGMP maintenance

\begin{tabular}{ll}
\hline Description & Cost \\
\hline Regulatory registration and lifecycle submission & $\$ 8000$ \\
Staff cGMP training & $\$ 5000$ \\
PQIT testing, stability test, media fills & $\$ 5000$ \\
Internal audits & $\$ 5000$ \\
Facility and equipment upkeep & $\$ 250,000$ \\
Total & $\$ 273,000$ \\
\hline
\end{tabular}


staff are re-qualified annually by a successful media fill simulation as part of the microbiology assurance.

Internal Audits Periodic audits are an essential element of the BMC's quality system for self-improvement. A typical 2-day audit by an external consultant costs approximately $\$ 5000$. As a cost-saving measure, during the same visit, the consultant is usually also contracted to provide annual cGMP staff training.

Facility and Equipment Upkeep The cost for maintaining a PET cGMP facility and equipment varies depending on the scope and nature of the maintenance. Preventive equipment maintenance, calibration, or constancy checks are done either in-house by well-trained and qualified chemists or contracted through a third party, either the original manufacturer or specialty service companies. Capital equipment, such as the cyclotrons, and complex analytical equipment, such as the GC, HPLC, and EndoSafe reader, are usually maintained through service contracts, which run at approximately $10 \%$ annually of the original equipment purchase value. If equipment undergoes major repair or other scenarios depicted in the quality system manual, the equipment will undergo performance re-qualification, and the related process or analytical method will be re-validated. We estimate the annual cost for facility and equipment maintenance cost is $\$ 250,000$ (synthesizer $\$ 30,000 /$ year, cyclotron approximately $\$ 220,000 /$ year).

\section{PET Drug Manufacturing Site Pro Forma Recommendations}

Based on the experience gained at UCLA in establishing a cGMP-compliant facility, the estimated costs for establishing a new cGMP-compliant PET drug manufacturing site are shown in Tables 3 and 4 . The revenue and costs of production are directly dependent on the PET drugs being produced. For the pro forma calculations outlined here, $\left[{ }^{18} \mathrm{~F}\right] \mathrm{FDG}$ is used as the PET drug.

Build-Out Expenses The one-time build-out costs in establishing a PET drug manufacturing site are shown in Table 3. More detailed explanations of the cost breakdowns are as follows.

Table 3. One-time build-out cost estimate for establishing a PET drug manufacturing site

\begin{tabular}{ll}
\hline One-time build-out expenses & Cost \\
\hline Cyclotron & $\$ 2,000,000$ \\
Hot cells & $\$ 500,000$ \\
Facilities build-out & $\$ 2,000,000$ \\
Initial equipment qualification cost & $\$ 300,000$ \\
ANDA application submission & $\$ 200,000$ \\
Total build-out cost & $\$ 5,000,000$ \\
\hline
\end{tabular}

Major Equipment and Related Costs Purchase of a cyclotron, along with two hot cells and two mini cells, are included, in addition to an estimated qualification cost for all the purchased equipment for the site.

Facilities An estimated new build-out cost of $\$ 2 \mathrm{M}$ is included. However, the cost can vary and be as low as $\$ 1 \mathrm{M}$ if retrofitting an existing facility. This dollar amount may vary significantly from one facility to another based on prior use of the facility and includes, but is not limited to, floor reinforcement, plumbing, ventilation and air conditioning, electrical power, and communication, as well as selective demolition and remodel/rebuilding to accommodate a cyclotron.

ANDA Application Submission Based on UCLA's experience submitting an ANDA application, we estimate a cost of $\$ 200,000$ for an external regulatory consulting firm to review, compile, and submit the application. The cost can vary significantly depending on the scope of support provided by the external organization and the level of internal resources utilized to prepare the ANDA application and related cGMP documents.

Ongoing Expenses and Revenue Table 4 outlines the recommended pro forma for a PET drug manufacturing site. The purpose of the pro forma is to demonstrate the cost and revenue associated with establishing a PET drug manufacturing business, and to estimate at which volume of dose production the facility can break even or make a profit. Of note, the cost of the build-out has not been added as part of the annual operating costs. The assumption is that the facility has made this as an upfront investment. If the facility chooses to finance aspects of the build-out, the costs should be added to the pro forma as an annual expense. The costs shown are estimated and extrapolated from UCLA's cGMP site business model.

A reimbursement rate of $\$ 145 /$ dose is estimated and used for $\left[{ }^{18} \mathrm{~F}\right] \mathrm{FDG}$. This amount is multiplied by the number of daily doses, resulting in the revenue numbers demonstrated in Table 4.

Non-personnel Costs The costs for QC supplies and consumables are expected to be constant for the BMC because the batch production, testing of $\left[{ }^{18} \mathrm{~F}\right] \mathrm{FDG}$, and ability to provide up to 50 doses can be performed with a single production. Production using kits might have a different cost model.

The UCLA BMC located at an academic medical school is not subject to paying rent to the university. This model may be different for private or other higher education institutions.

Synthesizer costs are estimated at $\$ 250,000 /$ synthesizer, depreciated over 7 years. We estimate that a single 
Table 4. Recommended pro forma for a PET drug manufacturing site

\begin{tabular}{|c|c|c|c|c|c|}
\hline & \multicolumn{5}{|l|}{ Daily dose } \\
\hline & 10 & 20 & 30 & 40 & 50 \\
\hline \multicolumn{6}{|l|}{ Annual OpEx—non-personnel cost } \\
\hline Production and quality control supplies and consumables & $\$ 74,100$ & $\$ 74,100$ & $\$ 74,100$ & $\$ 74,100$ & $\$ 74,100$ \\
\hline Rent and overhead & $\$ 0$ & $\$ 0$ & $\$ 0$ & $\$ 0$ & $\$ 0$ \\
\hline Synthesizers & $\$ 35,714$ & $\$ 35,714$ & $\$ 71,429$ & $\$ 71,429$ & $\$ 71,429$ \\
\hline Major production and $\mathrm{QC}$ equipment & $\$ 46,286$ & $\$ 46,286$ & $\$ 46,286$ & $\$ 46,286$ & $\$ 46,286$ \\
\hline Consulting support including staff GMP training & $\$ 50,000$ & $\$ 50,000$ & $\$ 50,000$ & $\$ 50,000$ & $\$ 50,000$ \\
\hline Cyclotron maintenance agreement & $\$ 180,000$ & $\$ 180,000$ & $\$ 180,000$ & $\$ 180,000$ & $\$ 180,000$ \\
\hline \multirow{2}{*}{$\begin{array}{l}\text { Maintenance/calibration agreements other than cyclotron (estimated at } 10 \% \text { of original } \\
\text { equipment purchase cost) }\end{array}$} & $\$ 32,400$ & $\$ 32,400$ & $\$ 32,400$ & $\$ 32,400$ & $\$ 32,400$ \\
\hline & $\$ 418,500$ & $\$ 418,500$ & $\$ 454,214$ & $\$ 454,214$ & $\$ 454,214$ \\
\hline \multicolumn{6}{|l|}{ Annual OpEx-personnel } \\
\hline One operations manager (salary \& benefits) & $\$ 180,000$ & $\$ 180,000$ & $\$ 180,000$ & $\$ 180,000$ & $\$ 180,000$ \\
\hline Two radiochemists ( $\$ 110,000$ salary \& benefits each) & $\$ 220,000$ & $\$ 220,000$ & $\$ 220,000$ & $\$ 220,000$ & $\$ 220,000$ \\
\hline One regulatory and quality assurance support & $\$ 120,000$ & $\$ 120,000$ & $\$ 120,000$ & $\$ 120,000$ & $\$ 120,000$ \\
\hline \multirow[t]{2}{*}{ Cyclotron engineer } & $\$ 0$ & $\$ 0$ & $\$ 0$ & $\$ 0$ & $\$ 0$ \\
\hline & $\$ 520,000$ & $\$ 520,000$ & $\$ 520,000$ & $\$ 520,000$ & $\$ 520,000$ \\
\hline Total annual operating costs & $\$ 938,500$ & $\$ 938,500$ & $\$ 974,214$ & $\$ 974,214$ & $\$ 974,214$ \\
\hline \multicolumn{6}{|l|}{ Annual revenue } \\
\hline FDG revenue at $\$ 145 /$ dose & $\$ 377,000$ & $\$ 754,000$ & $\$ 1,131,000$ & $\$ 1,508,000$ & $\$ 1,885,000$ \\
\hline Net revenue (excluding one-time build-out cost) & $(\$ 561,500)$ & $(\$ 184,500)$ & $\$ 156,786$ & $\$ 533,786$ & $\$ 910,786$ \\
\hline
\end{tabular}

synthesizer could supply up to 20 doses daily. A second synthesizer may be required to accommodate extra dose productions and a clinic dose schedule.

Major production and QC equipment costs are estimated at $\$ 320,000$, depreciated over 7 years.

Consulting support, including staff cGMP training, annual audits, and regulatory document submissions, is estimated at $\$ 50,000$.

The maintenance agreement for the cyclotron is estimated at $\$ 180,000$. This cost may vary depending on the level of support and services required.

The equipment maintenance agreement is calculated at $10 \%$ cost of the original equipment purchase (obtained suppliers of equipment). Based on an equipment cost of $\$ 320,000$, maintenance costs are estimated at $\$ 32,000$.

Personnel Costs Personnel costs are estimated at one operations manager, two radiochemists, and one dedicated regulatory support/quality assurance staff. Based on the $\mathrm{BMC}$ production, two radiochemists are needed for a single batch production. The personnel cost of production remains constant for 10-50 doses. This model may vary in other institutions, where one radiochemist may meet low dose production demands. The BMC does not utilize a cyclotron engineer and accounts for cyclotron maintenance and support through a cyclotron maintenance agreement instead.

\section{Discussion}

This article provides a framework for how the UCLA Department of Molecular \& Medical Pharmacology addressed the FDA requirements for PET cGMP.
As the PET cGMP enforcement date approached, academic PET manufacturing facilities chose from varying operational models based on their individual financial and operational constraints. Some academic sites opted to cease their production of PET drugs, which required regulatory filing and PET cGMP compliance. These sites are geographically close to commercial PET drug manufacturing sites and can depend on commercial sources for those PET drugs. In such instances, the radiochemistry facilities would be operated solely for preclinical studies or clinical researches under USP <823> Radiopharmaceuticals for Positron Emission Tomography (PET)_Compounding, Investigational, and Research Uses. A number of sites opted to partner with commercial radiopharmaceutical companies under the arrangement that they would be operating the academia-owned cyclotron and radiochemistry facility based on negotiated agreements. By doing so, these academic sites were relieved from the burden of PET drug regulatory submissions and cGMP compliance for clinical service.

Other academic sites, such as UCLA, decided to continue the PET drug manufacturing practice by establishing a PET cGMP environment and obtaining regulatory approvals. This decision was made in order to continue providing a flexible PET drug production schedule with an adequate number of PET drug doses to allow for a profitable operation. The net revenue from the NDA/ANDA PET drug production for the clinical service supports PET drug clinical research, as well as preclinical discovery and development at UCLA.

During the process of achieving and maintaining cGMP compliance, we have encountered inconsistent requirements for electronic audit trail capability. When proposing the final rule of PET cGMP, FDA opened it for public 
comment. One comment (comment \# 43) stated that "requiring the use of electronic audit trail software would be unduly burdensome for the PET community, and it recommended that we not require an electronic audit trail as part of PET cGMP provisions". FDA responded with "We agree that the additional level of quality assurance that might be provided through the use of electronic audit trail capability does not warrant the additional costs that would be imposed to implement this capability. Therefore the cGMP requirements for PET drug do not include electronic audit trail requirements" [2]. However, to our knowledge, based on the recent PET cGMP inspections, FDA inspectors require an electronic audit trail as part of data integrity requirements. The lack of electronic audit trail capability in the software was included in a number of FDA 483 inspectional observations.

Academic manufacturing sites have a relatively low production volume (one to two batches a day, each batch supplies several patient doses), and do not distribute the finished PET drugs commercially. Due to the financial, regulatory, and quality burden imposed on academic sites, we would like to suggest that the FDA take a balanced approach for cGMP compliance enforcement, to ensure the sustainability of academic sites, a driving force of PET innovation and development.

\section{Conclusion}

The University of California Los Angeles (UCLA) Biomedical Cyclotron (BMC) successfully converted from a precGMP era cyclotron and radiochemistry facility to a cGMP compliant PET drug manufacturer, with subsequent FDA approvals for two PET drug ANDAs. UCLA BMC established a sustainable cGMP-compliant operation model as an academic PET drug manufacturing site.

Acknowledgments. We thank Dr. Jorge Barrio for his expertise and advice and Ms. Bernadette Omote for providing editing and proofreading support to this manuscript.

\section{Compliance with Ethical Standards}

\section{Conflict of Interest}

The authors declare that they have no conflict of interest.

Open Access This article is distributed under the terms of the Creative Commons Attribution 4.0 International License (http:// creativecommons.org/licenses/by/4.0/), which permits unrestricted use, distribution, and reproduction in any medium, provided you give appropriate credit to the original author(s) and the source, provide a link to the Creative Commons license, and indicate if changes were made.

Publisher's Note. Springer Nature remains neutral with regard to jurisdictional claims in published maps and institutional affiliations.

\section{References}

1. Food and Drug Administration Modernization Act of 1997 (1997) 105th U.S. Congress. Retrieved from https://www.govinfo.gov/content/ pkg/PLAW-105publ115/html/PLAW-105publ115.htm. Accessed 19 Jun 2019

2. Current Good Manufacturing Practice for Positron Emission Tomography Drugs (Final rule) (2009) Federal Register vol 74, No. 236. Retrieved from https://www.gpo.gov/fdsys/pkg/FR-2009-12-10/pdf/E9-29285.pdf. Accessed 19 Jun 2019

3. Guidance: PET drugs - current Good Manufacturing Practice (CGMP) (small entity compliance guide) (2011) Food and Drug Administration. Retrieved from https://www.fda.gov/media/81038/download. Accessed 19 Jun 2019

4. Guidance: FDA oversight of PET drug products questions and answers (2012) Food and Drug Administration. Retrieved from https:// www.fda.gov/media/82911/download. Accessed 19 Jun 2019

Publisher's Note Springer Nature remains neutral with regard to jurisdictional claims in published maps and institutional affiliations. 